\title{
Nonlinear Transmission of a NOLM with a Variable Wave Retarder inside the Loop
}

\author{
Yazmin E. Bracamontes-Rodríguez ${ }^{1}$, Ivan Armas Rivera ${ }^{1}$, Georgina Beltrán-Pérez ${ }^{1}$ \\ J.Castillo-Mixcoátl $^{1}$, S.Muñoz-Aguirre ${ }^{1}$, Olivier Pottiez ${ }^{2}$, Baldemar Ibarra-Escamilla ${ }^{3}$, \\ Evgeny A. Kuzin ${ }^{3}$ \\ ${ }^{1}$ BUAP, Facultad de Ciencias Físico Matemáticas, Puebla, Mexico \\ ${ }^{2}$ Centro de Investigaciones en Óptica, León, Gto, Mexico \\ ${ }^{3}$ Instituto Nacional de Astrofísica, Óptica y Electrónica, Puebla, Mexico \\ yazmin_1985@yahoo.com.mx
}

\begin{abstract}
We research experimentally the configuration of a nonlinear optical loop mirror, with an imbalanced coupler, highly twisted low birefringence fiber and a variable wave retarder (VWR) inserted into the loop. The use of the variable wave retarder allows the easy adjustment of the nonlinear dependence of the NOLM transmission. The mechanism of VWR consists of applying an adjustable pressure on the optical fiber inside the loop to have different values of VWR through simple way. The nonlinear transmission of NOLM for different values of $\Delta$ and different amplitude of the input pulse were presented. The experimental results showed that VWR is a device that allows a simple adjustment of the nonlinear characteristic of the NOLM.
\end{abstract}

Keywords: nonlinear transmission, NOLM, variable wave retarder.

\section{Introduction}

Nonlinear Sagnac interferometer in optical fiber or nonlinear optical loop mirror (NOLM) device which was first introduced by N.J. Doran and David Wood (Doran N.J. y Wood D., 1998), is commonly used in nowadays in many applications, such as, optical switching (Moores J.D., et al., 1991; Cao W. et al., 2005; Agarwal, et al., 2005; Pottiez O. et al., 2004), wavelength the multiplexing (Sotobayashi H., et al., 2002), alloptical active mode locking (Tang W.W., et al., 2001), passive mode locking (Duling I.N., et al., 1991), pedestal suppression on pulses and pulse compression (Pelusi M.D., et al., 1999), and regeneration of ultrafast data streams (Sakamoto T. and Kikuchi K., 2005).

The setup of this device consist in a coupler whose output ports are fused by highly twisted fiber, which offer a versatile way to get nonlinear transmission or characteristic switching through Kerr effect, when a phase difference is present between two counterpropagating beams which interfere in the coupler. 
Most of the designs of these devices advantage the Self-Phase Modulation (SPM) effect, which cause a nonlinear phase difference, that is accumulated only, if appear asymmetry in the power between two counter-propagating beams in the loop, generally imposed by coupling ratio $(\alpha)$, which offers a very poor possibility to adjust the contrast or critical power and cannot have simultaneously high contrast and low critical power.

Other works report the use of a symmetrical coupler and a quarter-wave plate (QWP) retarder inside the loop near the coupler output port to generate a polarization asymmetry to obtain a high contrast and low critical power (Ibarra-Escamilla B., et al., 2004; Ibarra-Escamilla B., et al., 2005; Pottiez O., et al., 2005). The evolution of the nonlinear polarization (including Self-Phase Modulation (SPM) and Cross-phase modulation (XPM)), of the optical beam along the fiber it can be easily described using the coupling equations which was development by Kuzin (Kuzin E. A., et al., 2001). Many authors that work with optical fiber Sagnac interferometer obtain the characteristic transmission adapted to their particular applications, but usually give very few information about accurate manipulations required to achieve this result, therefore we decided to investigate an experimental study of the effect of a variable wave retarder (VWR) inside of the loop to obtain the easy adjustment of the nonlinear dependence of the NOLM transmission. For this case, the mechanism of VWR consists of applying an adjustable pressure on the optical fiber inside the loop to have different values of VWR through simple way. This mechanism is placed asymmetrically inside the loop with an imbalanced coupler $(\alpha=0.52$ and $\alpha=0.48)$. To close the loop a low birefringence optical fiber, highly twisted was used. A mode-locked fiber ring laser emitting pulses of $0.7 \mathrm{ps}$ duration with $1545 \mathrm{~nm}$ central wavelength was implemented as a source of signal.

The present work discusses the possibility of adjusting the nonlinear dependence of the NOLM by means of the polarization imbalance provoked by a VWR in the loop, which has not been reported up to date.

\section{Experimental Setup}

The experimental setup used to characterization of the NOLM is shown in fig. 1. We use a mode-locked fiber ring laser emitting pulses of 0.7 ps duration with $1545 \mathrm{~nm}$ central wavelength as a source of signal. The pulses were amplified by an EDFA with maximum amplification up to 20 times. The pulses from the amplifier output pass through a polarization controller PC, a polarizer, and a QWP. Rotation of the QWP allows generating a stable polarization state with desired ellipticity, in this particular case a circular polarization was used. After this, the pulses were splitted by a 90/10 coupler where the $10 \%$ is used to monitor the polarization and power of pulses in the input of NOLM.

The NOLM is formed by a slightly imbalanced 52/48 coupler, whose output ports were fusion-spliced with a 200-m loop of low-birefringence, highly twisted (6 turns $/ \mathrm{m}$ ) Corning SMF-28 standard fiber. A VWR retarder was created by applying an adjustable pressure on the optical fiber. Pressure produces a linear birefringence in this portion of the fiber with the slow axis in the direction that pressure is applied.

The VWR can be rotated to change the axes orientation. The retardation between the slow and fast axes can be varied between 0 and $2 \pi$ through pressure adjustment. The VWR was inserted near one of the output ports of the coupler. We performed 
measurement with the VWR inserted near both, the 0.52 port and the 0.48 port to compare the results. And finally the output pulses were detected by ultrafast photodetector with $8 \mathrm{GHz}$ and monitored by the oscilloscope with $2 \mathrm{GHz}$ of bandwidth. The transmission was calculated as a ratio between amplitudes of the output and input pulses.

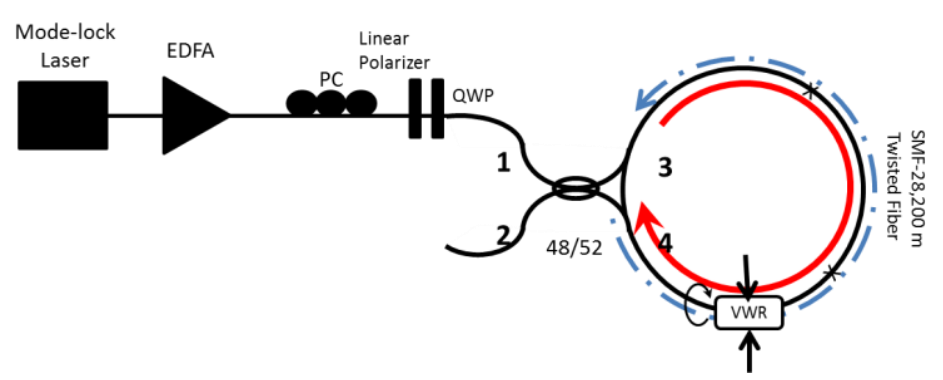

Fig. 1. Experimental setup.

\section{$3 \quad$ Results and Discussion}

Fig. 2, shows the result of measurements of NOLM transmission as a function of the amplitude of the input pulse for input ports, 0.52 and 0.48 , respectively. We can see the NOLM nonlinear transmission at $\Delta=0$ due to the power imbalance of the coupler. When the VWR is inserted to the 0.52 port the effect of the power imbalance adds to the effect of the polarization imbalance therefore the transmission grows with the increment of the retardation of the VWR. In contrast to the results shown in figure 2 (b), the polarization imbalance and power imbalance made opposite nonlinear phase shifts.

As a result at small retardations the transmission is less than for retardation equal to 0 . The transmission is 0 at any power at the retardation equal to $0.375^{*} \pi / 4$. For retardation higher than 0.375 the transmission starts to grow with the increment of the retardation. In the same figure we can note that occur a small increment of the low power transmission, less than $5 \%$. We attribute this to small misalignment of the VWR angle when the retardation is changed.

The complexity of the measurements of the transmission dependence on the pulse power arises from the fact that increasing of pulse power results in the same time the change of the other characteristics of the pulse such as pulse duration and chirp. These parameters also affect the NOLM transmission. To see clearly the effect of the VWR we measured the transmission against the retardation at the constant pulse power. Fig. 3 , shows the nonlinear transmission of the NOLM as a function of the VWR for different input pulse amplitudes when the VWP is inserted to the 0.52 port.

When the pulse amplitude has a value of 1.43 , the dependence data at pulse power of $70 \mathrm{~W}$, except that the minimum value occurs when $\Delta=0.8$. This shift may be due to not very exact calibration of the VWR or because the input polarization was not exactly circular. 


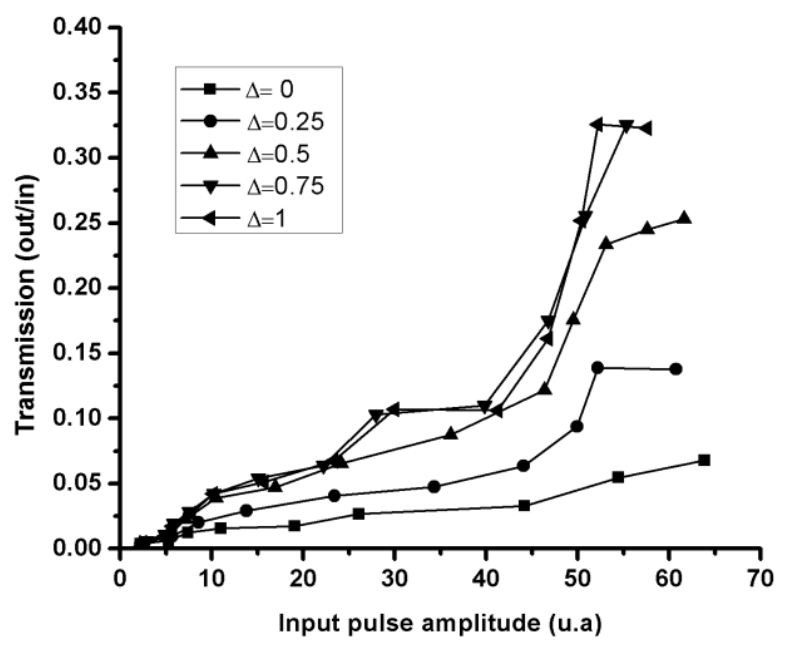

a)

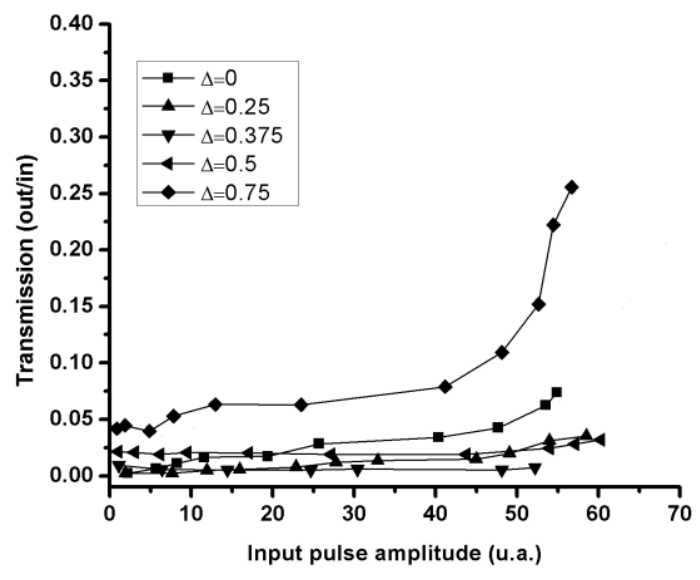

b)

Fig. 2. Transmission of NOLM versus input pulse amplitude, (a) input port of $52 \%$ and (b) input port of $48 \%$.

Fig. 4 Show that the transmission reaches to a maximum when $\Delta=1.2$. Another important feature is that when $\Delta<0.5$ there is a minimum of transmission since it is considered that the nonlinear effects are subtracted because the difference powers between the counter propagating beams in the NOLM. 


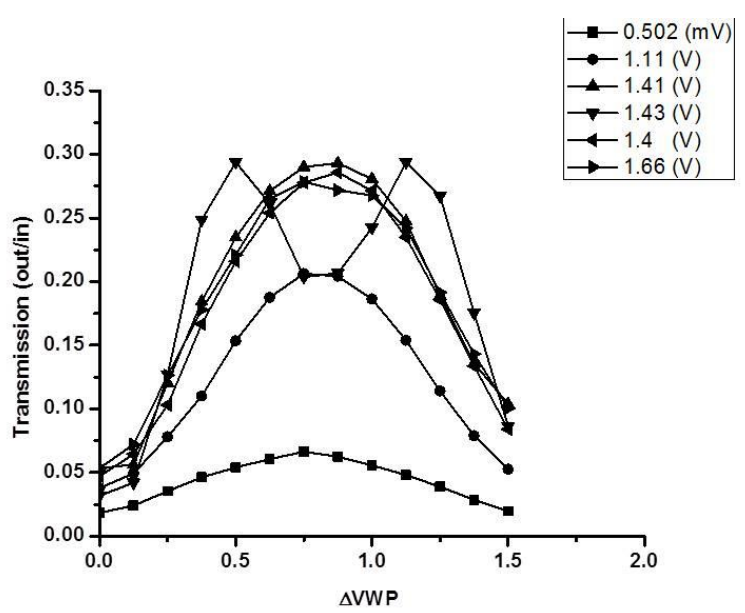

Fig. 3. Transmission of NOLM versus different values of $\triangle \mathrm{VWP}$, experimental results input port of $\alpha=0.52$.

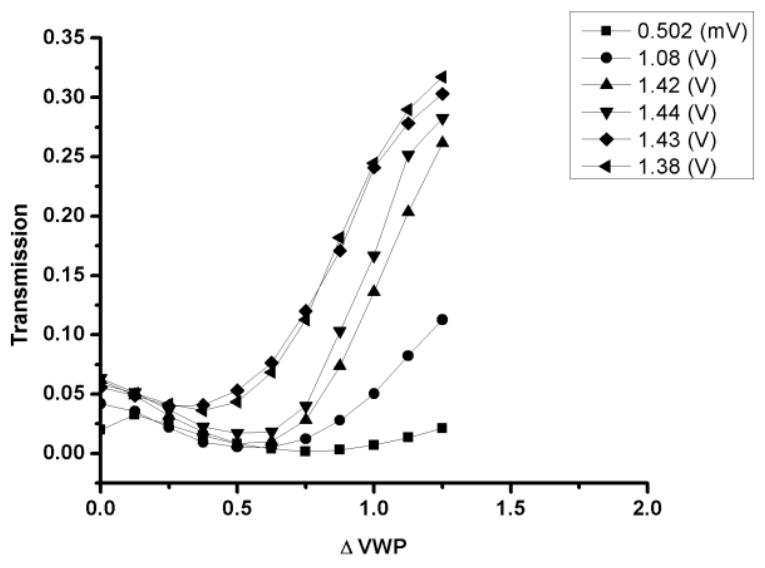

Fig. 4. Transmission of NOLM versus different values of $\square$ VWP, experimental results of input port of $\alpha=0.48$.

\section{Conclusions}

In this work an experimental study of nonlinear transmission of NOLM for different values of $\Delta$ and different amplitude of the input pulse were presented. A simple and new device as a variable wave retarder was used inside of the loop. That the experimental results showed that VWR is a device that allows a simple adjustment of the nonlinear characteristic of the NOLM. 


\section{References}

1. Agarwal, A., Wang, L., Su, Y., Kumar, P.: All optical loadable and erasable storage buffer based on parametric nonlinearity in fiber. J. Lightwave Technol., 23, pp. 2229-2238 (2005)

2. Cao, W., Wai, P. K. A.: Comparison of fiber-based Sagnac interferometers for self forSwitching of optical pulses. Opt. Commun., 245, pp. 177-186 (2005)

3. Doran, N. J., Wood, D.: Nonlinear optical loop mirror. Opt. Lett., 13, pp. 56-58 (1998)

4. Duling, I. N.: All- fiber ring soliton laser mode locked with a nonlinear mirror. Opt. Lett., 16, pp. 539-541 (1991)

5. Ibarra-Escamilla, B., Kuzin, E., Pottiez, O., Haus, J., Gutierrez-Zainos, F., GrajalesCoutiño, R., Zaca-Moran, P.: Fiber optical loop mirror with a symetrical coupler and quarter-wave retarder plate in the loop. Opt. Commun, 242, pp. 191-197 (2004)

6. Ibarra-Escamilla, B., Kuzin, E., Zaca-Moran, P., Grajales-Coutiño, R., MendezMartinez, F., Pottiez, O., Roja-Laguna, R., Haus, J. W.: Experimental investigation of the nonlinear optical loop mirror with twisted fiber and birefrigence bias. Optics Express., Vol 13, 26, pp. 10760-10767 (2005)

7. Kuzin, E. A., Korneev, N., Haus, J. W., Ibarra-Escamilla, B.: Theory of nonlinear loop mirrors with twisted low-birefringence fiber. J. Opt. Soc. Am. B, 18, pp. 919-925 (2001)

8. Moores, J. D., Bergman, K., Haus, H. A., Ippen, E. P.: Optical switching using fiber ring reflectors. J. Opt. Soc. Am. B, 8, pp. 594-601(1991)

9. Pelusi, M. D., Matsui, Y., Suzuki, A.: Pedestal suppression from compressed femtosecond pulses using a nonlinear fiber loop mirror. IEEE J. Quantum Electron, 35, pp. 867-874 (1999)

10. Pottiez, O., Kuzin, E. A., Ibarra-Escamilla, B., Camas-Anzueto, J. L., GutierrezZainos, F.: Experimental demostration of NOLM Switching bassed on nonlinear polarization rotation. Electron. Lett., 40, pp. 892-894 (2004)

11. Pottiez, O., Kuzin, E. A., Ibarra, B., Mendez-Martinez, F.: Theorical investigation of the NOLM with highly twisted fibre and a $\chi / 4$ birrefringence bias. Opt. Commun., 254, pp. 152-167 (2005)

12. Sakamoto, T., Kikuchi, K.: $160 \mathrm{GHz}$ operation of nonlinear optical loop-mirror with an optical bias controller. IEEE Photonics Technol. Lett., pp. 543-545 (2005)

13. Sotobayashi, H., Sawaguchi, C., Koyamada, Y., Chujo, W.: Ultrafast walk-off-free nolineal optical loop mirror by a simplified configuration for $320 \mathrm{Gbit} / \mathrm{s}$ time-division multiplexing signal demultiplexing. Opt. Lett., 27, pp. 1555-1557 (2002)

14. Tang, W. W., Shu, C., Lee, K. L.: Rational harmonic mode locking of an optically triggered fiber laser incorporating a nonlinear optical loop modulator. IEEE Photon Technol. Lett., 13, pp. 16-18 (2001) 